\title{
HACIA UN PERFIL PROFESIONAL DEL TRADUCTOR EN COLOMBIA
}

\section{TOWARDS A DESCRIPTION OF THE GOLOMBIAN TRANSLATOR}

FECHA DE RECEPCIÓN: 14 de enero FECHA DE APROBACIÓN:10 de marzo Pp. 42-57
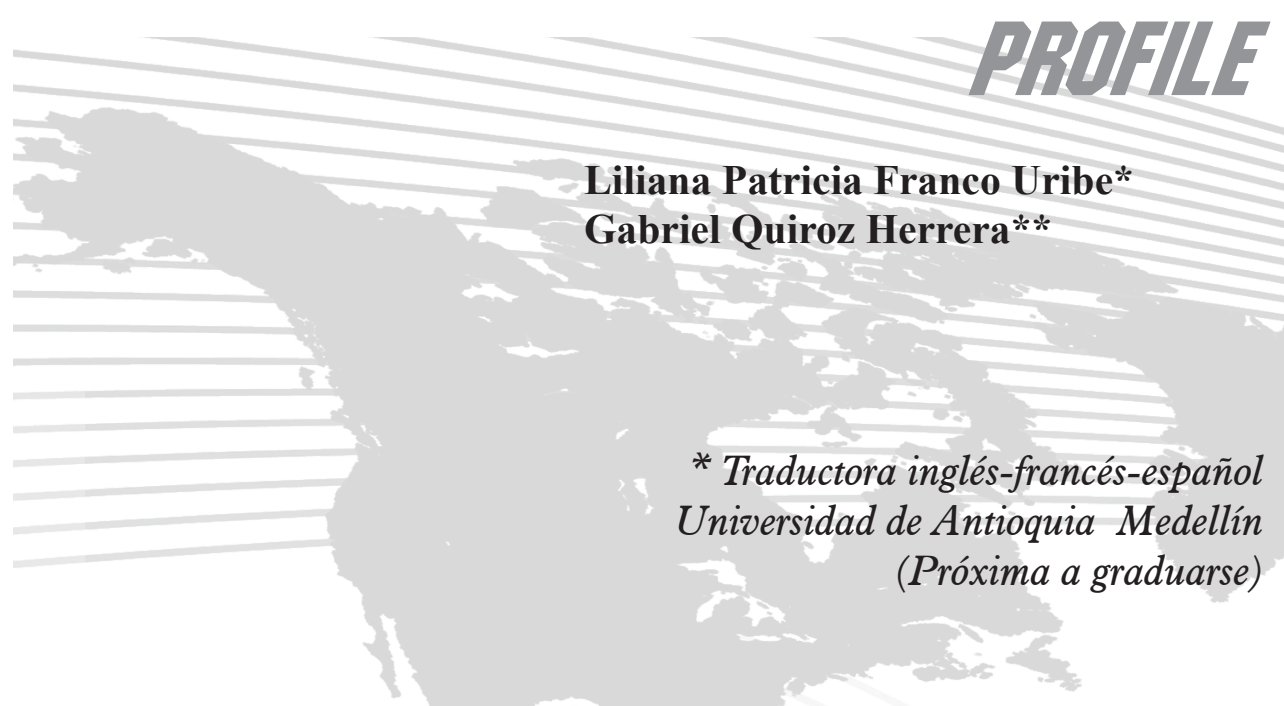

*Doctor in Applied Linguistics (2008). Pompeu Fabra University Institute for Applied Linguistics (IULA)-Spain. Thesis entitled:

Los sintagmas nominales extensos especializados en inglés y en español:

Descripción y clasificación sintáctica y semántica en un corpus de genoma. Supervisor: Prof. Dr. Mercè Lorente.

Grade: "Excelent Cum Laudem"

Postgraduate Diploma in Translation

(Especialista en Traducción) (1997).

University of Antioquia School of Languages-Colombia.

Thesis entitled: La traducción de textos científico-técnicos hacia lengua extranjera por medio de rastreos terminológicos (The Translation of Scientific and Technical Texts into a Foreign Language Using Terminology Research). Supervisor: Prof. Dr. Cecilia Plested Grade: "Good”. Associate Professor and Researcher Head of the Translation Program School of Languages, University of Antioquia. 


\section{RESUMEN}

En este artículo, se presentan los resultados de una investigación en la que se buscaba determinar el perfil del traductor en Colombia. Para ello, se empleó una encuesta a la cual respondieron 45 traductores consultados. El perfil del traductor en Colombia se resume como un profesional de entre 36 y 45 años de edad, que vive y trabaja principalmente en Medellín o Bogotá, con estudios universitarios de pregrado y posgrado en traducción, que se desempeña como traductor independiente entre tiempo completo y medio tiempo del inglés al español y que emplea herramientas informáticas.
$\Rightarrow$ Palabras claves

Traducción

Estatus del traductor

Aspectos profesionales de la traducción

Traducción en Colombia

Perfil del traductor

\section{ABSTRACT}

In this article, the results of a research to establish the translator's profile in Colombia are presented. A survey was sent to 57 translator and 45 answered it. This profile of the Colombian translator can be drawn as a professional aged 36 to 45, living and working in Medellin and Bogotá with undergraduate and graduate degree mainly in translation, working as freelance translator full and part time from English into Spanish, and using computer tools.

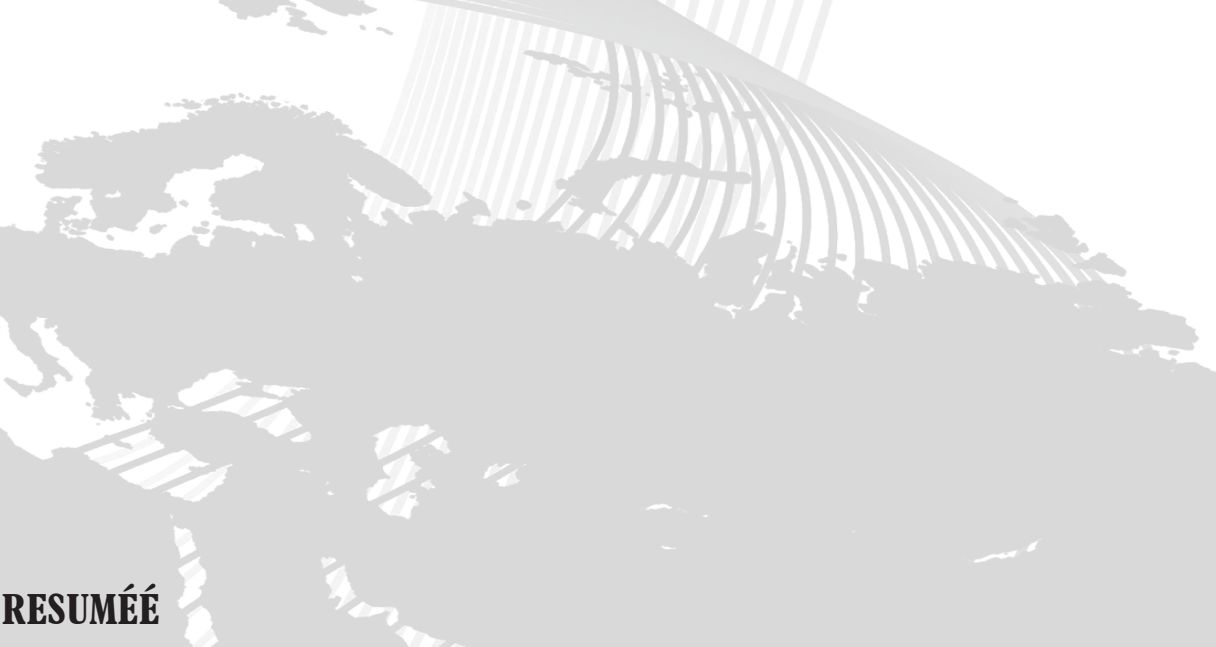

Cet article présente les résultats d'une recherche à travers la quelle nous avons essayé de déterminer le profil du traducteur en Colombie. À cet effet nous avons employé un questionnaire et réalisé une enquête à laquelle ont répondu 45 traducteurs. Le profil du traducteur en Colombie peut se résumer comme étant un professionnel ayant entre 35 et 45 ans, qui vit et travaille principalement à Medellín ou Bogota, qui a réalisé des études universitaires de premier et second cycle, principalement dans le domaine de la traduction, qui travaille comme traducteur indépendant à temps complet ou à mitemps, qui effectue des traductions de l'Anglais à l'Espagnol, et qui emploie des outils informatiques.
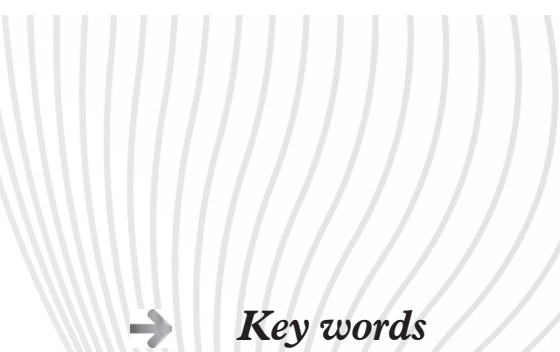

Key words

Translation

Translator status

Professional aspects of translation

Translation in Colombia Translator's profile

\section{$\Rightarrow \quad$ Mots clefs \\ Traduction \\ Statut du traducteur \\ Aspects professionnels de la traduction \\ Traduction en Colombie \\ Profil du traducteur}




\section{INTRODUCción}

Buando los españoles llegaron a América en 1492, se encontraron con un escenario multilingüe sin precedentes: más de 1.000 lenguas indígenas entre ellas azteca, maya, paéz, chibcha, caribe, tupi guaraní, aymará, quechua entre otras. Como lo señala Bastin $(1998 ; 505)$, la necesidad de interpretes en la región era obvia. Sin embargo, existe muy poca evidencia sobre este aspecto. Por razones históricas bien conocidas, la América Latina de lengua española es prácticamente una región monolingüe aunque muchas lenguas indígenas aún subsisten. En Colombia, la constitución política de 1992 reconoció el derecho histórico de las comunidades indígenas a su territorio, lengua y cultura. Desde entonces, somos un país plurilingüe en teoría, pero en la práctica la situación ha cambiado muy poco.

Como lo propone Quiroz (2002: 3), podría argumentarse entonces que las necesidades de servicios lingüísticos como la traducción, la interpretación y la terminología en Colombia son obvias. Sin embargo, nada más lejos de la realidad. Por eso, en Colombia el estudio y la práctica de estas disciplinas de manera sistemática son relativamente nuevos aunque durante muchos años han existido traductores e intérpretes.

Ya que en Colombia la traducción como profesión es una actividad muy reciente, esta no alcanza a tener un reconocimiento en la sociedad como una actividad laboral o profesional, sino como un hobby o como un ejercicio paralelo a otras áreas del conocimiento, incluida la propia lingüística.

Desde un punto de vista legal, la profesión de traductor e intérprete está regida en parte, desde 1951 por el Decreto 382 sobre el examen para el ejercicio del oficio de traductor e intérprete oficial y diferentes artículos de leyes y decretos desde entonces, hasta el Decreto 962 de la Ley anti-trámites de 2005 sobre la calificación de los traductores oficiales y las instituciones que pueden convocar al examen.

Desde el punto de vista académico, en Colombia se dicta un sólo pregrado de traducción en la Universidad de Antioquia, dos maestrías en la Universidad Autónoma de Manizales y la Universidad EAN y, desde 1995, se han dictado varias especializaciones de posgrado (universidades de Antioquia, Valle, Nacional, Rosario, Nariño y Jorge Tadeo Lozano). Aunque son varios los grupos de investigación que se interesan en esta materia, no son siquiera comparables con la gran cantidad de grupos en medicina, ingenierías y otras carreras profesionales que se ofrecen en el país.

Desde el punto de vista profesional, el único avance, aunque muy significativo, hecho en 2010 es la aprobación por parte del Comité Técnico 218 -Terminología y otros recursos lingüísticos y de contenido- de Icontec de la norma técnica colombiana 5808: Servicios de traducción. Requisitos para la prestación del servicio.

Desde el punto de vista gremial, existen en Colombia dos cuerpos colegiados: la Asociación Colombiana de Traductores e Intérpretes-ACTI- y el Colegio Colombiano de Traductores-CCT-; entidades que trabajan por el posicionamiento de la traducción como profesión en Colombia y mantienen una agenda muy activa para lograrlo.

Por tanto, en este artículo se presentan los resultados de una investigación llevada a cabo en 2008 en la cual se pretendía conocer el perfil del traductor en Colombia, es decir, quiénes traducen, en qué ciudades viven, cuáles son sus estudios superiores, qué herramientas manejan, entre otras particularidades.

Partiendo de estos datos, se intenta eliminar algunos interrogantes que tiene la sociedad sobre la traducción, en aras de posicionarla como una carrera profesional importante para el desarrollo del país como cualquier otra profesión.

Como resultado se obtuvo un conjunto de referencias que permitieron esbozar un perfil inicial sobre los traductores en Colombia, abriéndole paso a nuevas investigaciones sobre el tema y sobre el mercado actual y potencial de la traducción. 


\section{JUSTIFICACIÓN}

Tal y como lo establecen Dam y Zethsen (2010: 194), las investigaciones sobre el estatus del traductor tanto en sus aspectos legales, sociales y profesionales es una de las áreas menos estudiada en los estudios de traducción. Dicha afirmación, se hace en el marco de los países europeos en los cual se presenta el mayor desarrollo de la disciplina tanto en el ámbito académico como profesional.

Son pocos los estudios acerca de la traducción en Colombia. Entre ellos, se encuentra el "Estudio de mercados Programa de Traducción" (Flórez et al.) realizado por la Facultad de Ciencias Económicas de la Universidad de Antioquia, en el año 1999. Debido a que este estudio se realizó hace 10 años, ya no tiene la misma relevancia para el mercado actual debido a que los cambios de la profesión han sido considerables en esta década. Por otra parte, la Asociación Colombiana de Traductores e Intérpretes (ACTI) cuenta, en su página Web, con una encuesta que tiene como propósito crear una base de datos que proporcione soluciones a las necesidades tanto de traductores como de usuarios de la traducción, partiendo del perfil del traductor. Sin embargo, no ha arrojado ninguna clase de resultados abiertos al público, de hecho, la encuesta ha estado disponible por varios meses y aún no se ha cerrado.

Igualmente, Clavijo et al (2006) presentan un estudio sobre el perfil del traductor pero desde la perspectiva de la empresa. En este estudio se presenta un perfil en el cual no se tiene en cuenta la condición o estatus del traductor sino que se le ve como una necesidad perfilada por las empresas y sólo se realiza en Bogotá con lo cual la muestra no es representativa pues hay muchas empresas en Medellín, Cali, Barranquilla, Cartagena que pueden emplear traductores y su sede social se encuentra en estas ciudades. Aún así, el estudio nos muestra también una realidad del estado del traductor en Colombia y concuerda en algunos aspectos con este estudio como veremos más adelante.

En definitiva, se desconoce cualquier información vigente sobre el perfil del traductor, su mercado y su actividad laboral desde la perspectiva de los mismos traductores. Por ende, el propósito de este artículo es definir cuál es el perfil del traductor en Colombia para resolver el interrogante generalizado sobre quienes traducen en este país.

En Colombia la traducción como profesión es una actividad muy reciente, esta no alcanza a tener un reconocimiento en la sociedad como una actividad laboral o profesional, sino como un hobby o como un ejercicio paralelo a otras áreas del conocimiento, incluida la propia lingüistica. 
artiendo de que la traducción en Colombia es una labor informal para la cual no se necesita tener ninguna profesionalización, ni estudio especifico, inició la búsqueda del perfil del traductor en otros lugares del mundo en los cuales la traducción cobra mayor importancia.

\subsection{Perfil europeo}

Según el Libro blanco de la traducción en España (1997, 14), Ginebra fue una de las ciudades que creó uno de los primeros centros de formación de traductores e intérpretes, durante la II Guerra Mundial (1941).

Fue sólo hasta 1990 que la traducción ingresó al grupo de carreras profesionales con una duración de 4 años, por lo que se empezaron a crear pregrados y postgrados enfocados a la actividad traductiva. Hoy en día, España cuenta con unas 20 universidades con facultad de traducción e interpretación, entre las que podemos mencionar a Granada, UAB, Palma de Gran Canaria y Salamanca.

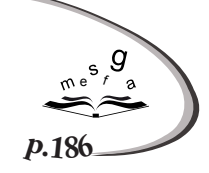

Gracias a este aumento de ofertas de estudio y a la necesidad de ofrecer traductores idóneos, Europa creó la norma de calidad UNE-EN 15038: 2006, específica para servicios de traducción ${ }^{1}$.

El perfil del traductor europeo se divide en dos enfoques (2002:31). En primer lugar, está el enfoque de las relaciones internacionales, es decir, traductores interesados en la mediación interpersonal, la redacción y revisión de textos y la gestión de información multilingüe. Dicho profesional trabajaría para instituciones y empresas internacionales (tales como empresas de turismo), en las cuales se enfrentaría a traducciones generales e interpretación.

En segundo lugar, está el enfoque especializado. Los traductores interesados en traducción especializada, terminología e interpretación de conferencias entrarían en esta categoría. Dichos profesionales se enfrentarían a traducciones jurídicas,

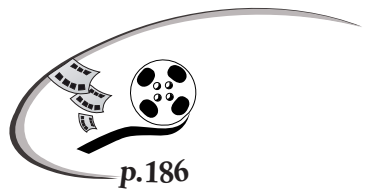
audiovisuales, técnicas y literarias.

\subsection{Perfil canadiense}

Durante la década de los 60 y los 70, Canadá entró en la industria de la traducción; al mismo tiempo aparecieron los primeros profesionales y, para la década de los 80 , surgían las primeras empresas de traducción.

Un estudio de mercadeo sobre la industria de la traducción en Canadá, realizado en 1997, reveló que a la fecha existían 804 empresas de traducción, terminología e interpretación y estimó que había aproximadamente 4.500 traductores independientes.

Debido a que Canadá cuenta con dos idiomas oficiales, los traductores generalmente tienen un perfil orientado hacia el francés y el inglés; sin embargo, el par de lenguas que más se traducía para 1997 era inglés-español. Tal era la oferta que alrededor del $80 \%$ de los traductores vivían sólo de la traducción y su mayor cliente era el sector público.

Aunque este estudio se realizó hace más de 10 años, sus datos son alentadores, pues arrojan cifras que confirman porqué Canadá es uno de los países en los que la traducción es una labor profesional bien posicionada en el mercado.

\subsection{Perfil colombiano}

A pesar de que la sociedad colombiana tiene una necesidad bien clara del oficio del traductor y del intérprete al ser un país monolingüe en la práctica y tener bajos niveles de lengua extranjera entre su población, y que existe una figura legal del traductor e intérprete oficial, no existe una

\footnotetext{
${ }^{1}$ En Colombia es la NTC 5808: 2010 de Icontec modificada.
} 
ley que le dé al traductor un reconocimiento profesional más allá de la figura legal para asuntos diplomáticos y judiciales. Colombia, a diferencia de otros países latinoamericanos, no tiene una legislación para el oficio de la traducción o la interpretación, todo se reduce a unos pocos artículos en los que se define la función de perito y traductor oficial: Testigo de lengua extranjera, Artículo 427. Documentos procedentes del extranjero, Artículo 428. Traducción de documentos) consagrados en el Código de procedimiento penal y una disposición del congreso (Ley 962 de 2005- Art. 33 la cual es una modificación del artículo $4^{\circ}$ del decreto 382 de 1951.) que habla del examen para el ejercicio del oficio de traductor e intérprete oficial.

Desde un punto de vista histórico, según Orozco (2000: 73) los escritores colombianos durante el siglo XIX podían darse el lujo de traducir literatura por simple placer y "además de ser intelectuales y poliglotas, esos escritores se dedicaron a la traducción".

Sin embargo, las necesidades del mundo moderno exigen una labor traductiva mayor, por la cual es necesario ofrecer una carrera que forme traductores profesionales competentes, que puedan traducir por placer, pero que además puedan ganarse la vida con dicha labor.
El único estudio de fácil acceso es el "Estudio de mercados Programa de Traducción". No obstante, este estudio confirma que existe un bajo porcentaje de profesionales en traducción vinculados a empresas, debilidad que podría ser causada por la poca difusión y el poco mercadeo de la traducción.

Según Clavijo et al. (2006: 73), el perfil del traductor desde una perspectiva de la empresa se resume a continuación:

"Algunos de los resultados expuestos anteriormente, permiten concluir que, en general, el traductor freelance es requerido en casos puntuales, pues la mayor parte de la comunicación en otras lenguas es manejada y realizada internamente en la empresa; y quien actúa como traductor in-house es un miembro de la empresa que conoce las dos lenguas, aunque no siempre tiene especialización en el área correspondiente. La mayor demanda actual en traducción es escrita, en mayor

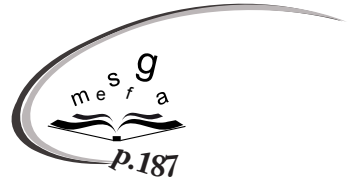
volumen de inglés a español que de español a inglés y los directores de departamentos y coordinadores de área son quienes más solicitan estos servicios."

Puede ver así, después de esta breve presentación de perfiles y si se compara la información de Colombia con Canadá Europa, podríamos concluir que esta carrera profesional apenas está empezando a desarrollarse.

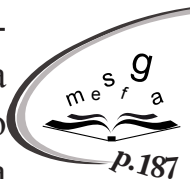

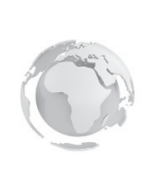

\section{METOdOLOGÍA}

El instrumento empleado en la metodología para desarrollar esta investigación fue la encuesta. A continuación se enumeran de forma más detallada la metodología empleada durante la investigación.
Las necesidades del mundo moderno exigen una labor traductiva mayor, por la cual es necesario ofrecer una carrera que forme traductores profesionales competentes, que puedan traducir por placer, pero que además puedan ganarse la vida con dicha labor. 


\subsection{Redacción de la encuesta}

El primer paso a realizar fue la selección de los criterios necesarios para crear el perfil de traductor: edad, estudios superiores, idiomas que aplica, tiempo de experiencia, tipos y formatos de textos que traduce y herramientas que maneja. Luego se especificaron otros criterios importantes a la hora de analizar el mercado en el cual se desempeñaban: ciudad de residencia, trabajador independiente o asalariado, certificados profesionales y pertenencia o no a una asociación de traducción. Finalmente y con el fin de organizar la encuesta para no olvidar ningún detalle o dato importante, se crearon cuatro grandes categorías que servirían para estructurar la encuesta y categorizar la información recolectada. Las categorías fueron: datos personales, formación profesional, información laboral y aspectos profesionales.

Luego de tener las categorías claras y los criterios que pertenecían a cada una de ellas, se crearon preguntas abiertas, preguntas cerradas conúnica respuesta y preguntas cerradas con múltiples respuestas. La única categoría con preguntas de respuesta opcional fue la categoría de datos personales para evitar que los participantes no contestaran la encuesta al sentir intromisión en sus datos personales.

\subsection{Distribución de la encuesta}

Como el objetivo principal era conocer el perfil del traductor colombiano, fue necesario que la encuesta estuviera publicada de modo que pudiera llegar a todos los traductores sin importar su lugar de residencia. Para ello, se escogió un recurso de creación de encuestas online llamado Survey Monkey.

\subsection{Recolección de datos}

Survey Monkey, como se dijo anteriormente, fue la herramienta para la distribución y recolección de la encuesta. Esta página crea la encuesta, la distribuye y por último arroja los resultados completamente tabulados, siempre y cuando sean preguntas cerradas.

Al finalizar la recolección de datos se pudo establecer con claridad cual era la muestra de análisis como se detalla abajo. Las siguientes son algunas definiciones necesarias antes de pasar al análisis de los datos.

\subsection{Muestra}

La muestra de esta investigación se compone de 57 traductores que realizan su actividad traductiva en Colombia, a los cuales se les envió la encuesta por medio de correo electrónico. Si se tiene en cuenta estudios realizados en países como Dinamarca con un mercado bien establecido en los cuales hay una muestra de 244 traductores (131 traductores freelance), podemos afirmar que la muestra en nuestro estudio es bien relevante si tenemos en cuenta que el contexto colombiano está formado por traductores freelance. Para la muestra de análisis respondieron la encuesta 45 traductores.

\section{RESULTADOS}

A continuación, se realiza el análisis de las preguntas, agrupándolas y relacionándolas entre sí. Además, se presentan los diagramas correspondientes para cada caso. Es importante, aclarar que no es necesario analizar todas las preguntas, en especial las que corresponden a la categoría de datos personales; por lo tanto, sólo se analizarán las que sean pertinentes. 


\section{Pregunta 1: nombre}

Sólo 28 encuestados respondieron esta pregunta (62,8\%). Así, de acuerdo a los nombres, podemos afirmar que 15 son sexo masculino y 13 son de sexo femenino. Aún así, no es posible afirmar si existen más mujeres que hombres en la profesión pues tampoco fue una variable a tener en cuenta durante el trabajo.

FIGURA 1. Distribución por la edad

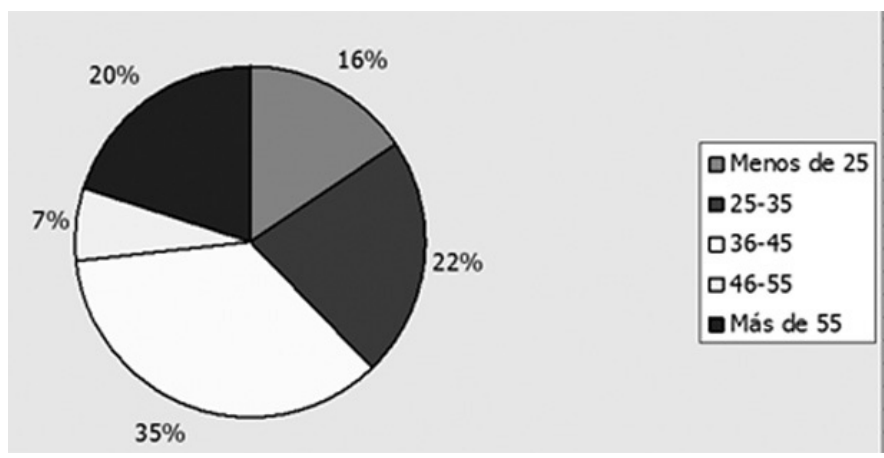

Fuente. Los autores

\section{Pregunta 3: nacionalidad}

En esta respuesta, puede observar que el 93\% de los encuestados son colombianos y el 6,7\% de otras nacionalidades (Reino Unido, Holanda e Italia). Así, si tomamos en cuenta que los idiomas que más se traducen según la pregunta 14 son el inglés y el español, y que la traducción

\section{Pregunta 2: edad}

El 35\% de los traductores se encuentra entre los 36 y los 45 años de edad y le siguen los traductores entre 25 y 35 años con un $22 \%$. Esta respuesta cobra mucho sentido al relacionarla con la pregunta 7 y 8 acerca de los estudios realizados. Podría pensarse que entre mayor edad, mayor cantidad de estudios especializados; sin embargo, son respuestas aisladas que sólo generan simples suposiciones. directa es la que más se realiza según la pregunta 15 , podemos deducir que el traductor colombiano traduce generalmente del inglés a su lengua materna el español. A continuación, se presentan los diagramas de las respuestas 3 y 15.
FIGURA 2. Distribución por nacionalidad

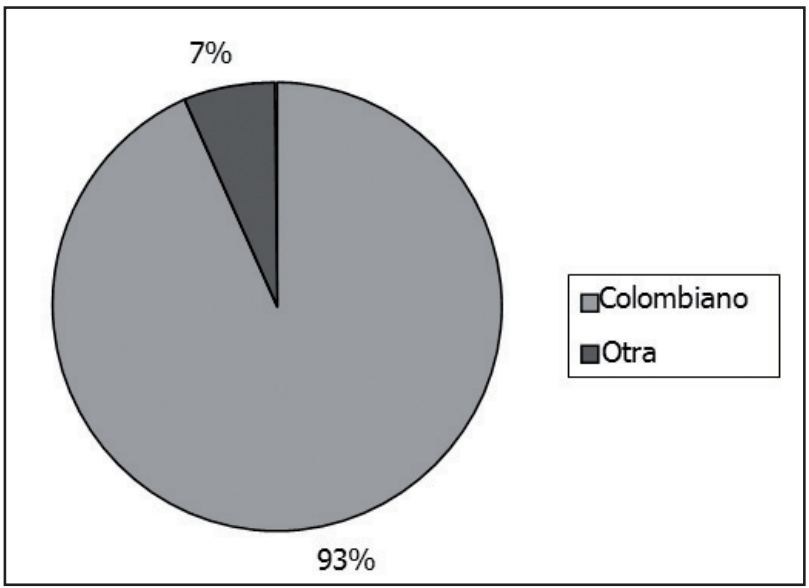

Fuente. Los autores
FIGURA 3. Dirección de la traducción

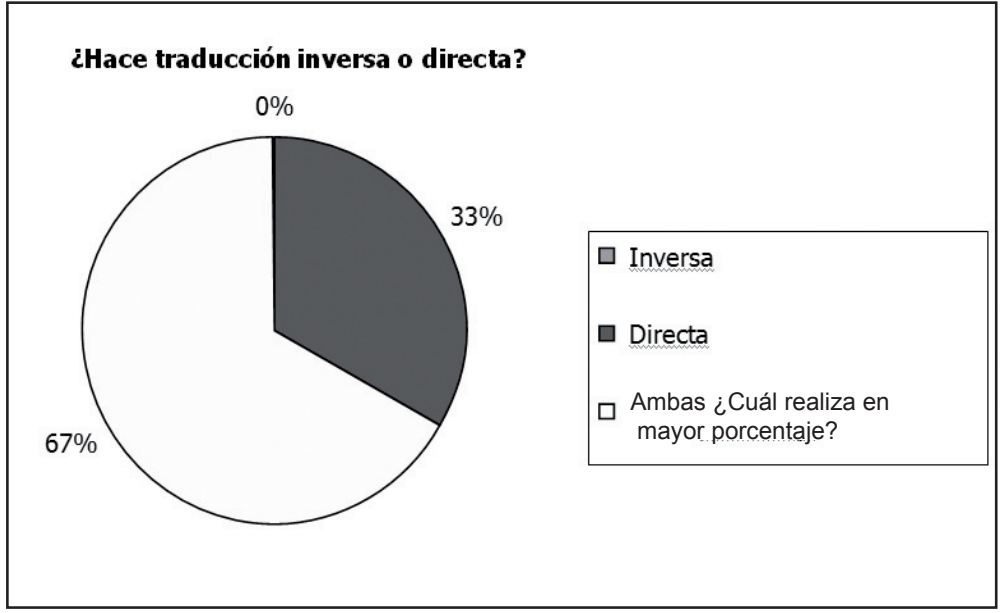

Fuente. Los autores 


\section{Pregunta 4: ciudad y departamento \\ de residencia}

El fin de esta pregunta es saber donde viven los traductores en Colombia.

Los datos demuestran que Antioquia es el departamento con mayor número de traductores (71\%), seguido por Bogotá $(13,3 \%)$, Estos no son totalmente confiables debido a que los contactos de los autores, ambos habitantes de ciudades de Antioquia, también viven en Antioquia. Es importante destacar que, aún así, se contactaron a los traductores en todas las zonas de país y de las dos asociaciones, ACTI y CCT que tienen su sede en Bogotá. De todos modos, puede observarse en los datos que los traductores se ubican en los centros de producción industrial (Medellín, Bogotá y Cali) y de enseñanza de la traducción (Medellín, Bogotá y Cali).

FIGURA 4. Distribución por lugar y residencia

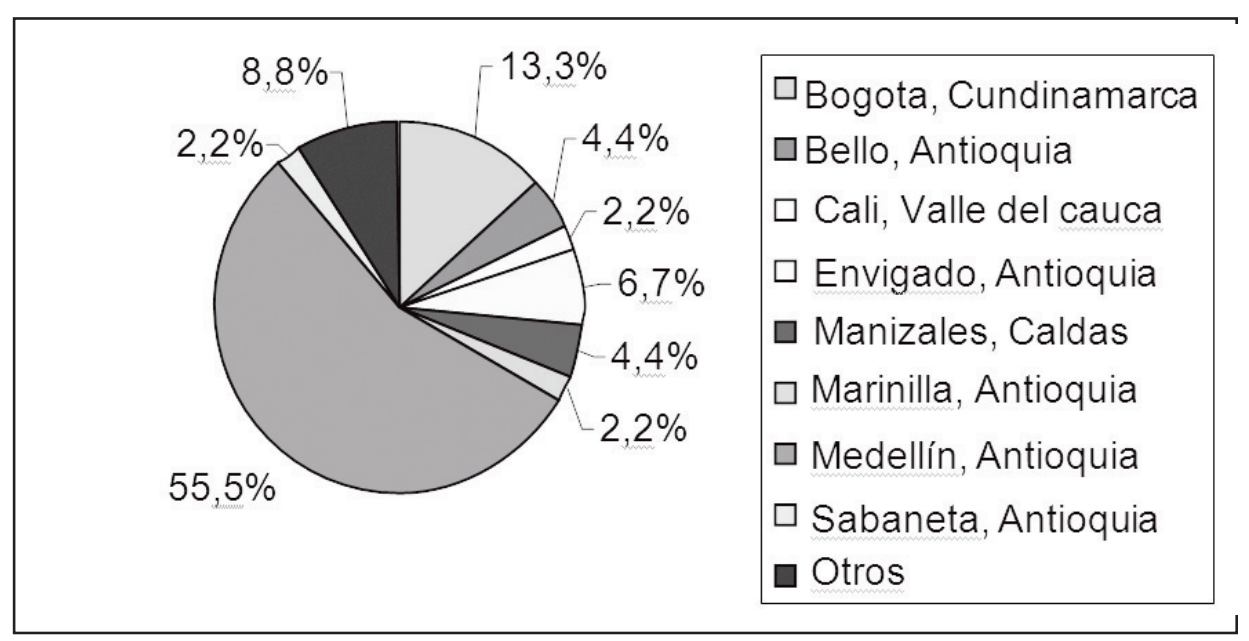

Fuente. Los autores

\section{Pregunta 7: ¿Qué estudio(s) de pregrado ha realizado?}

Este es una de los resultados más alentadores. Más de la mitad de los traductores ha estudiado traducción e interpretación $(48,9 \%$ y 2,2\%). El resto de encuestados estudió carreras afines a las lenguas (licenciatura en lenguas modernas, filología y lenguas y secretariado bilingüe) y un porcentaje relativamente bajo se alejó totalmente del tema medicina, ingeniería ambiental, artes y periodismo. Solo una persona respondió que no ha realizado ningún estudio de pregrado.

FIGURA 5. Distribución por formación académica en pregrado

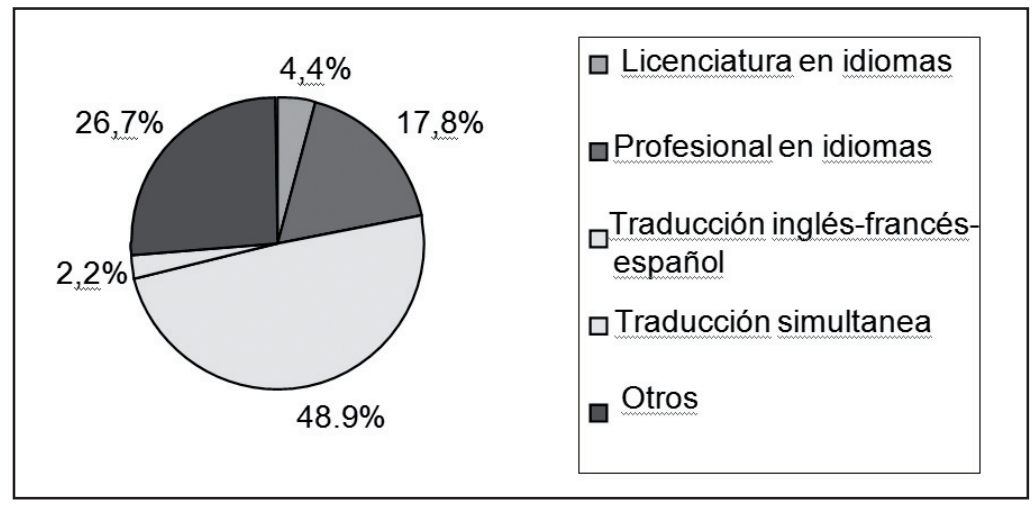




\section{Pregunta 8: ¿Ha realizado algún(os) estudio(s) de postgrado?}

Continuando con los resultados positivos, la mayoría de los encuestados ha realizado estudios de posgrado y maestrías (un 55,6\%), por lo general, en traducción o áreas relacionadas con la lingüística aplicada, periodismo, letras, filosofía y derecho.

FIGURA 6: Distribución por formación académica en posgrado

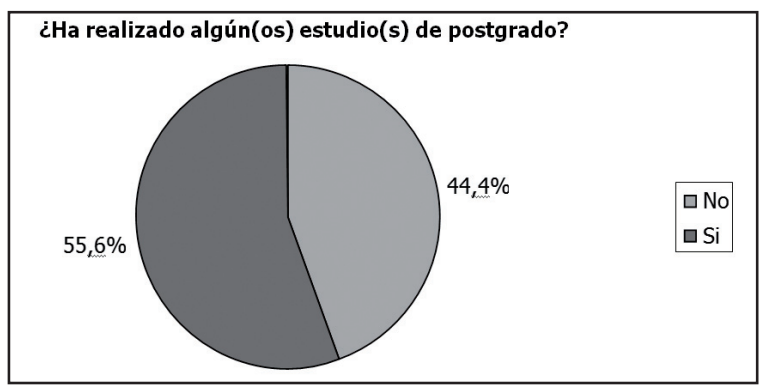

Fuente. Los autores

\section{Pregunta 9: ¿Ha realizado algún otro tipo de estudio(s)?}

La mayoría de los traductores en Colombia han realizado estudios de pregrado y de postgrado, pero no han realizado estudios adicionales $(55,6 \%)$. Sin embargo, los porcentajes están muy parejos y, por su parte, la minoría si ha realizado cursos y seminarios ya sea sobre traducción, música, incendios, etc. (44,4\%).Estos cursos adicionales son importantes porque los traductores deben tener una cultura general muy amplia y si es posible, especializarse en un tema específico. En este sentido, Clavijo et al. (2006: 73) muestran que las empresas colombianas prefieren a un traductor idóneo para realizar sus traducciones.

\section{FIGURA 7: Distribución por formación continuada}

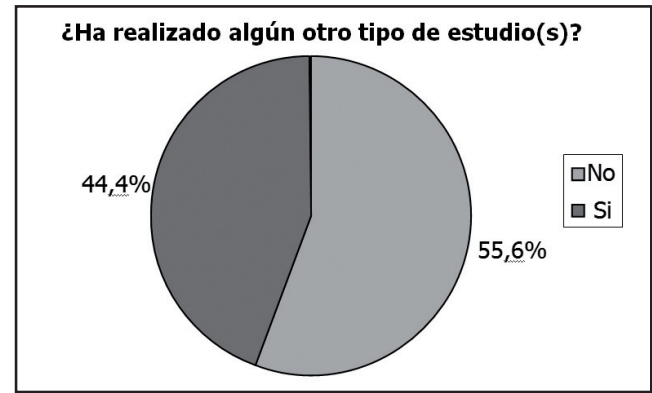

Fuente. Los autores

\section{Pregunta 10: ¿Tiene algún certificado profesional?}

Esta pregunta demuestra el poco interés de los traductores por especializarse y por unir fuerzas para posicionar el mercado de la traducción. Lastimosamente, el 73,3\% de los traductores en Colombia no tienen ningún certificado profesional, no solo por la falta de interés, sino por la gran cantidad de requisitos necesarios para obtener uno, sin mencionar los precios que se deben pagar.

FIGURA 8: Distribución por certificación

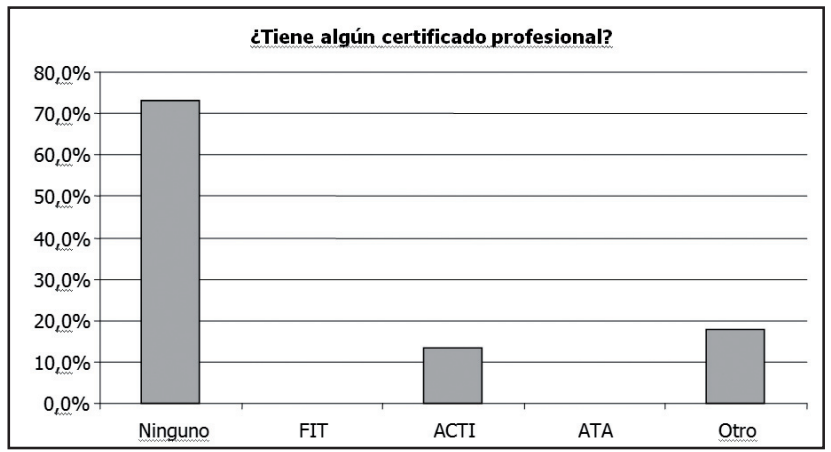

Fuente. Los autores

\section{Pregunta 11: ¿Es trabajador independiente 0 asalariado?}

Puesto que, en Colombia, la traducción como profesión es una profesión en desarrollo, la existencia de agencias y empresas de traducción de pequeño tamaño que contraten traductores es casi nula. Por lo anterior, los resultados de esta pregunta son obvios: la mayoría de los traductores en Colombia son independientes $(60 \%)$ y siguen la tendencia de muchos países como Dinamarca en donde más del 50\% son traductores independientes (Dam y Zethsen 2010: 194) y en Colombia las empresas emplean más a traductores de este tipo que a agencias (Clavijo 2006: 73).

FIGURA 9: Distribución por tipo de contrato

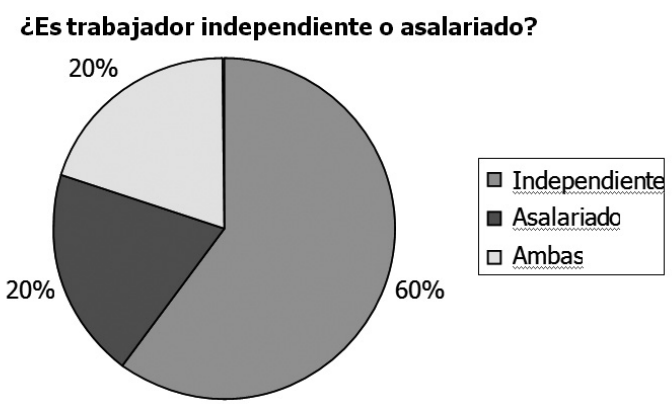

Fuente. Los autores 


\section{Pregunta 12: ¿Hace cuántos años es traductor?}

Según los datos, el 36\% de los traductores en Colombia cuentan con más de 10 años de experiencia en la profesión, seguido de un $18 \%$ entre 8 y 10 años. Esta respuesta tiene sentido, ya que los primeros años de experiencia corresponden al tiempo en el que el traductor realiza parte de su pregrado, los siguientes corresponde al tiempo que usa durante la maestría o postgrado y, finalmente, el tiempo que continúen ejerciendo su labor. Este resultado se correlaciona con la edad ya que el $36 \%$ tiene entre 35 y 45 años de edad y con lo expuesto antes. Clavijo et al. (2006: 72) muestran que las empresas prefieren a los traductores con buena experiencia como un factor de selección.

FIGURA 10: Distribución por antigüedad

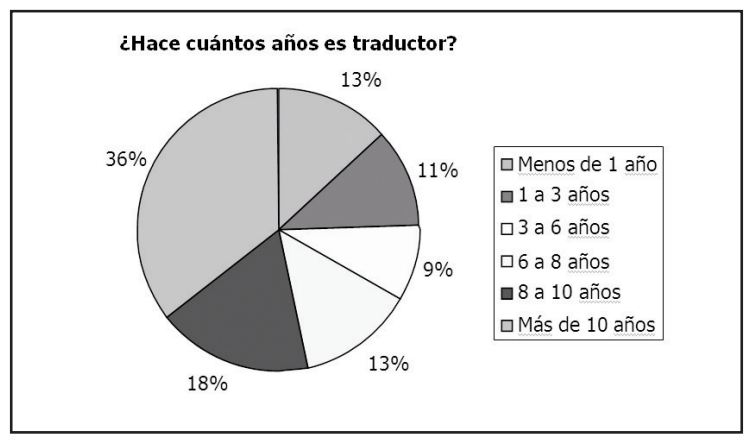

Fuente. Los autores

\section{Pregunta 13: ¿Cuánto tiempo dedica a traducir?}

La mayoría de los traductores en Colombia, aparte de ser independientes, solo trabajan ocasionalmente. Solo el $24 \%$ se dedican a tiempo completo a la traducción, $13,3 \%$ a medio tiempo y un $37,8 \%$ trabajan ocasionalmente en traducción.

Si además sumamos los datos obtenidos en la respuesta 18 (un $75,6 \%$ realizan otra actividad: docentes, secretarios, músicos profesionales, estudiantes, ganaderos o vendedores), llegamos a la conclusión de que es necesario combinar la traducción con otro tipo de actividad profesional para poder alcanzar la carga laboral necesaria para subsistir o debido a oportunidades laborales o gustos en el ejercicio profesional. A continuación, se presentan tanto los datos de esta pregunta como los datos de la pregunta 18 , ya que estas van de la mano.
FIGURA 11: Distribución por tiempo de traducción

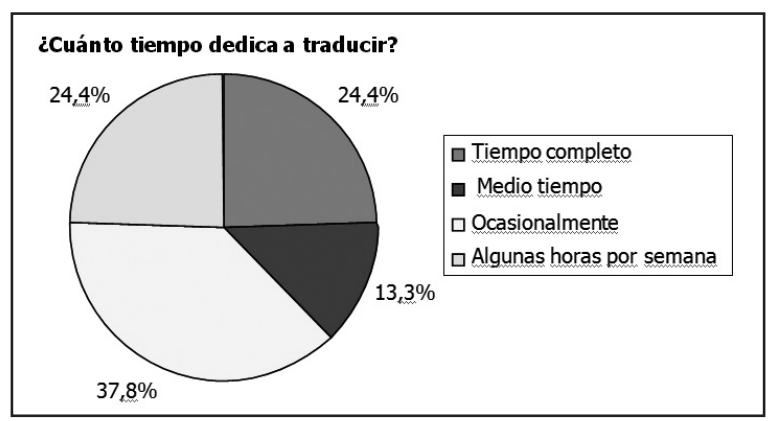

Fuente. Los autores

FIGURA 12: Distribución por otras actividades laborales

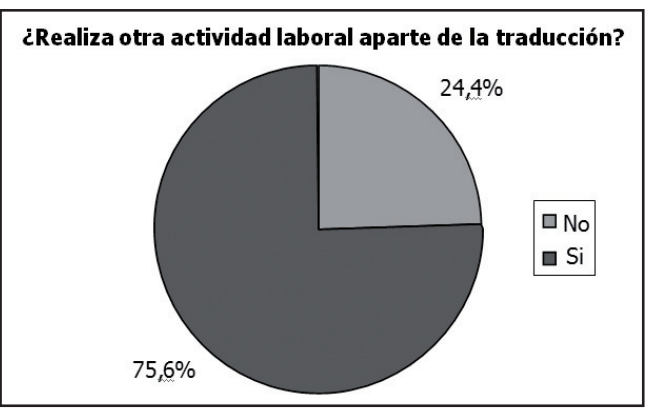

Fuente. Los autores

\section{Pregunta 14: ¿Qué idiomas trabaja?}

Como es de esperarse, el inglés es el idioma más traducido en Colombia (88,9\%), seguido por el francés $(62,2 \%)$. Los datos arrojados por esta pregunta son totalmente claros y lógicos, ya que son los idiomas que más se han acercado a Colombia, ya sea por la música, el comercio o la cultura en general. Esta encuesta concuerda en lenguas con la realizada por Zielinski, D. y Ramírez (2005), Clavijo et al. (2006: 73). Igualmente, los traductores en Colombia, traducen sólo hacia su lengua materna un $33,3 \%$ y un $66,7 \%$ hacen traducción directa e inversa.

FIGURA 13: Distribución por lenguas de trabajo

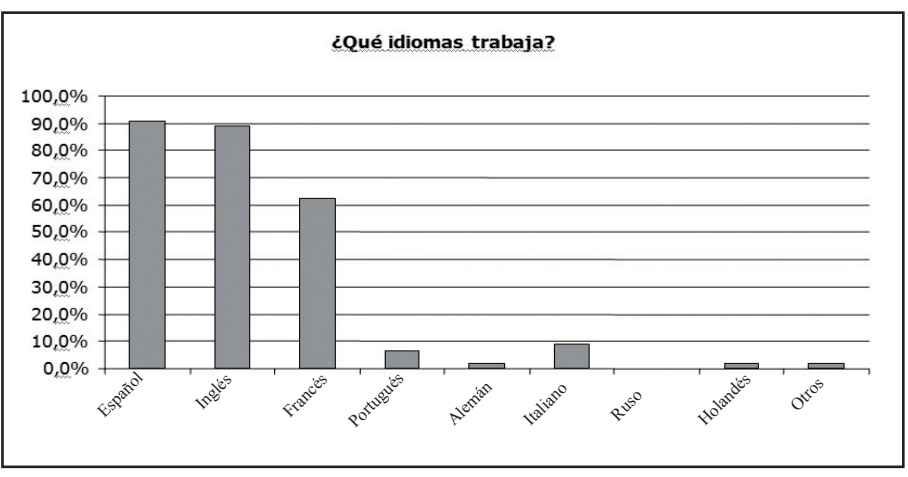

Fuente. los autores 


\section{Pregunta 16: ¿Qué tipo de traducciones realiza?}

En esta pregunta, que es de respuesta múltiple, los datos establecen que la mayoría de los traductores en Colombia realizan traducciones técnicas $(84,4 \%)$, seguidas por traducciones científicas $(68,9 \%)$. Ahora bien, si analizamos la pregunta 17, observamos que el tipo de traducción que se realiza con mayor frecuencia es la traducción técnica y, en este caso, la científica comparte el mismo porcentaje con la traducción jurídica. Esta encuesta concuerda en con el tipo de traducción realizada por Lagoudaki (2006: 12). Obsérvese que nuevos tipos de traducción comienzan a emerger como un tipo de traducción habitual, tales como la traducción audiovisual (20\%), la localización (20\%) y adaptación (20\%). En cambio, otros tipos de traducción más tradicionales en Colombia como la traducción literaria $(31,1 \%)$ y la traducción oficial $(31,1 \%)$, aunque permanecen, ya no son el eje principal de la actividad profesional entre los traductores profesionales.

Igualmente, puede observarse que, aunque la traducción técnica y científica son el eje profesional de los traductores en Colombia, hay una variedad importante de tipos de traducción, lo que muestra que poco a poco el mercado comienza a crecer.

FIGURA 14: Distribución por tipo de traducción

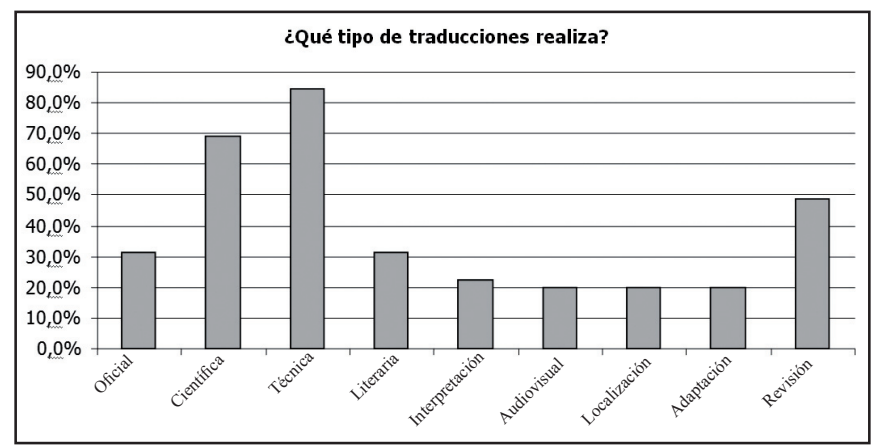

Fuente. Los autores

FIGURA 15: Distribución por frecuencia por tipo de traducción

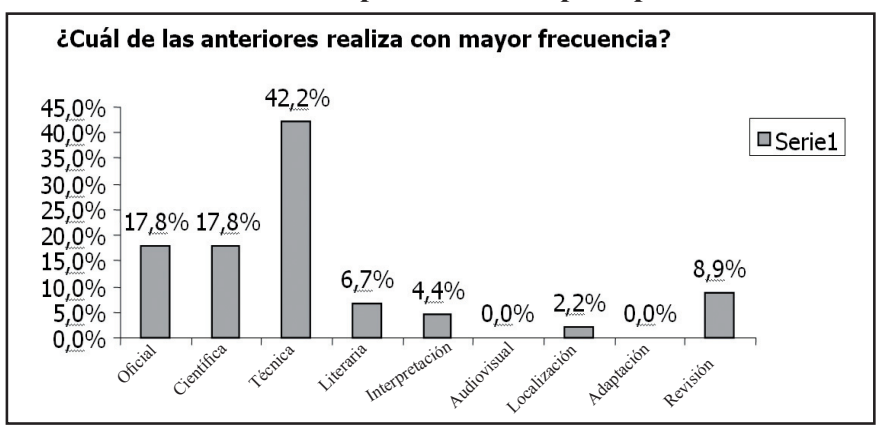

Fuente. Los autores

\section{Pregunta 19: ¿Cómo califica su competencia con el computador?}

Tener un buen conocimiento y manejo del computador es totalmente importante para los traductores para sobrevivir en el mercado actual. Los traductores consideran que tienen un manejo excelente $(31,1 \%)$ y bueno del computador $(62,2 \%)$ para más de un $90 \%$.

Además, si tenemos en cuenta la pregunta 20, puede verse que los formatos que más se traducen son los de Office: Word, Excel, PowerPoint, etc. con un 90\%, luego los archivos en formato PDF con un $62 \%$. Internet también es una herramienta que les permite a los traductores realizar su labor de una manera más cómoda, pues cuentan con foros, bases de datos terminológicas, corpus y diccionarios especializados, todo desde la comodidad de su casa.

Es importante, resaltar que, a pesar de toda la revolución informática, aún un 50\% de los traductores en Colombia también trabajan con papel. En este sentido, el mercado colombiano sigue a la retaguardia ya que en estudios europeos, los textos en papel solo representan un 19\% (Lagoudaki. 2006: 12).

En conclusión, la mayoría de los traductores en Colombia tienen buenas competencias con el computador y, por lo general, la mayoría traduce formatos de MS Office.

FIGURA 16: Distribución por competencia con el computador

Fuente. Los autores

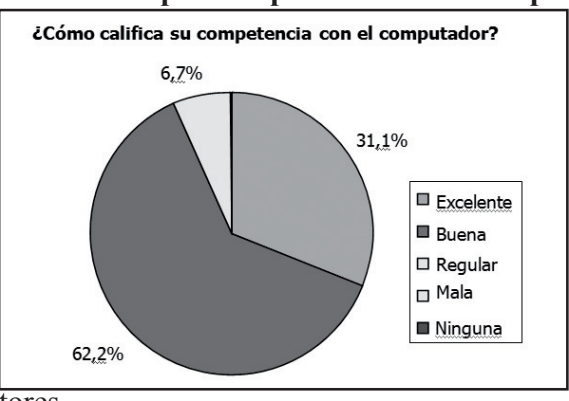

FIGURA 17: Tipos de formatos

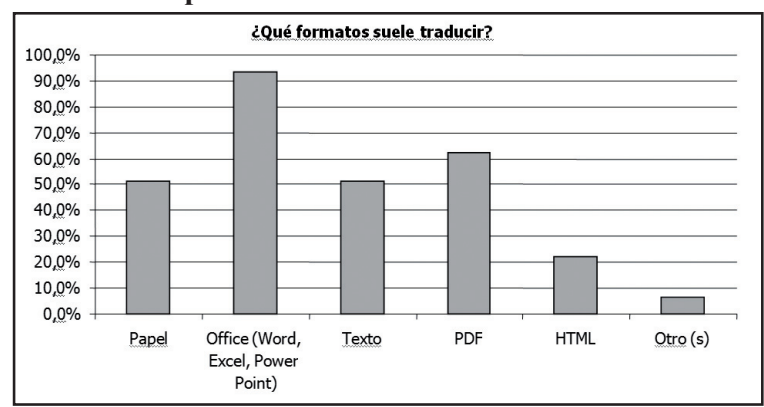

Fuente. Los autores 


\section{Pregunta 21: ¿Con cuál (es) herramienta (s) de traducción trabaja?}

Los datos demuestran que la mayoría de los traductores en Colombia traducen con Transit (35,6\%), seguida de Trados con un $24,4 \%$ y SDL $4,4 \%$ (de la misma casa) para un total de $32,4 \%$. Igualmente, podemos observar que existe entre los traductores colombianos una variedad importante de herramientas TAC (Similis, MemoQ, Across, Bitrext, Oli-fant, Rainbow, entre otras). Según la encuesta tan sólo el 28,9\% de los traductores no utilizan ninguna herramienta para traducir, porcentaje bastante similar al de los encuestados que no estudiaron una carrera profesional afín con lo idiomas $(26,7 \%)$. Es totalmente posible que quienes no estudiaron carreras profesionales afines con los idiomas, nunca hayan tenido contacto o información sobre las herramientas utilizadas para traducir, por lo cual no las usan.

\section{FIGURA 18: Distribución por tipo de herramienta TAC}

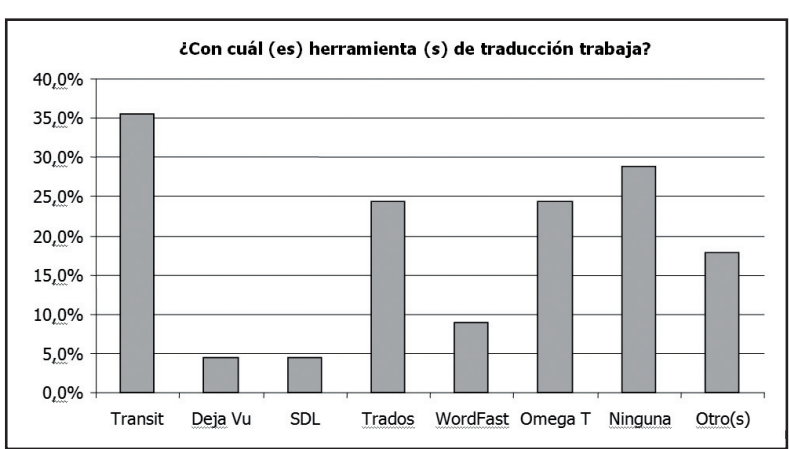

Fuente. Los autores

\section{Pregunta 22: ¿Pertenece a alguna asociación de traductores?}

Como se comentó antes, en Colombia no existe una cultura traductiva o al menos quienes traducen no se animan a conformar asociaciones o a crear grupos para posicionar a la traducción en el mercado. Parece que los traductores no tienen sentido gremial y prefieren trabajar solos, en lugar de intentar trascender en el mercado.

Los datos muestran que el $75,6 \%$ de los traductores en Colombia no hacen parte de ninguna asociación de traductores.
FIGURA 19: Distribución por asociación profesional

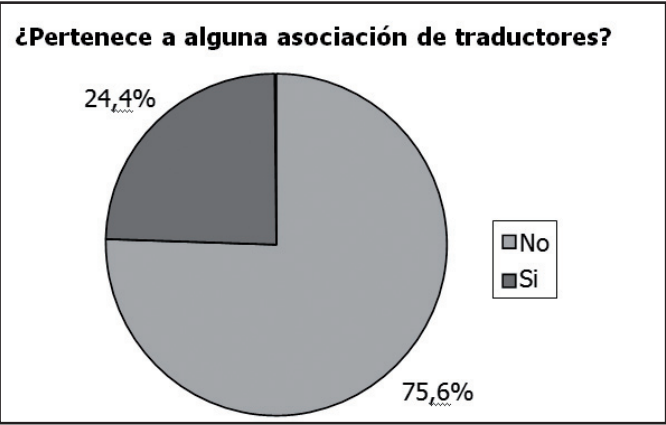

Fuente. Los autores

\section{Pregunta 23: ¿Cómo cobra una traducción?}

Esta es una pregunta polémica y tabú dentro de la profesión en Colombia y son las asociaciones las que tienen una idea más clara del asunto. Los profesionales europeos cobran con base en el texto de partida y los programas TAC así lo hacen para entregar las estadísticas al usuario. Sin embargo, muchos traductores en Colombia insisten en cobrar con base en el texto de llegada. Según los datos, la mayoría de los traductores en Colombia cobra con base en el texto de partida, siguiendo con los criterios europeos $(66,7 \%)$. Aún así, el porcentaje es bajo de acuerdo con los parámetros internacionales. En este sentido la NTC 5808 (14), recomienda cobrar con base en la lengua de partida y hace recomendaciones en este aspecto.

FIGURA 20: Distribución por método de cobro de acuerdo a la lengua

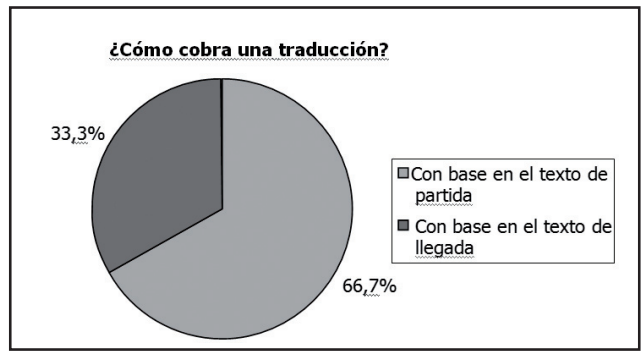

Fuente. Los autores

\section{Pregunta 24: ¿En qué parámetro se basa para fijar el precio de una traducción?}

Esta pregunta, aunque menos polémica, también cobra mucha importancia entre los traductores. ¿Es correcto cobrar por página? o ¿es mejor cobrar por palabra? En realidad, todo depende del tipo de traducción que se vaya 
a hacer, como lo plantea la misma NTC 5808 (2010: 14). Un ejemplo de ello, sería recibir una traducción en papel imposible de escanear o digitalizar; en este caso nadie contaría las palabras, simplemente cobraría por página. Sin embargo cuando se trata de estandarizar, dejando a un lado los inconvenientes, la mayoría de traductores en Colombia prefieren cobrar por palabra $(57,8 \%)$ aunque los que cobran por página siguen siendo muchos también $(33,3 \%)$.

FIGURA 21: Distribución por método de cobro

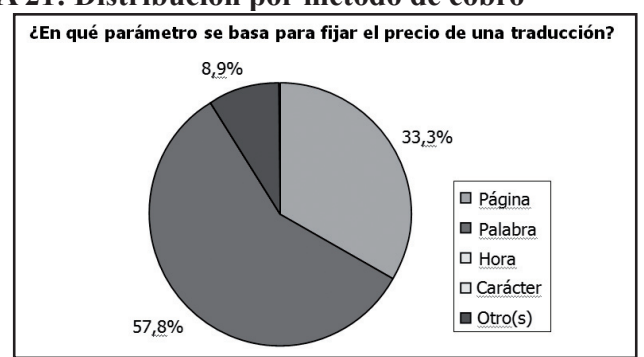

Fuente. Los autores

\section{Pregunta 25: ¿Qué medios utiliza para promocionar sus servicios?}

Debido a la falta de asociaciones, sociedades y agrupaciones, los traductores en Colombia en su mayoría promocionan sus servicios por medio de contactos personales $(86,7 \%)$. Este resultado es la consecuencia de una cantidad de factores económicos, sociales y culturales que existen en Colombia respecto a la traducción, entre ellos, todos los mencionados en las preguntas anteriores. Igualmente, puede decirse que los traductores no suelen promocionar sus servicios adecuadamente mediante el uso de las nuevas tecnologías. Incluso, los métodos tradicionales como avisos y páginas amarillas no son bien utilizados (un 4,4\% y 15,6\%, respectivamente).

FIGURA 22: Distribución por método de promoción de servicios de traducción

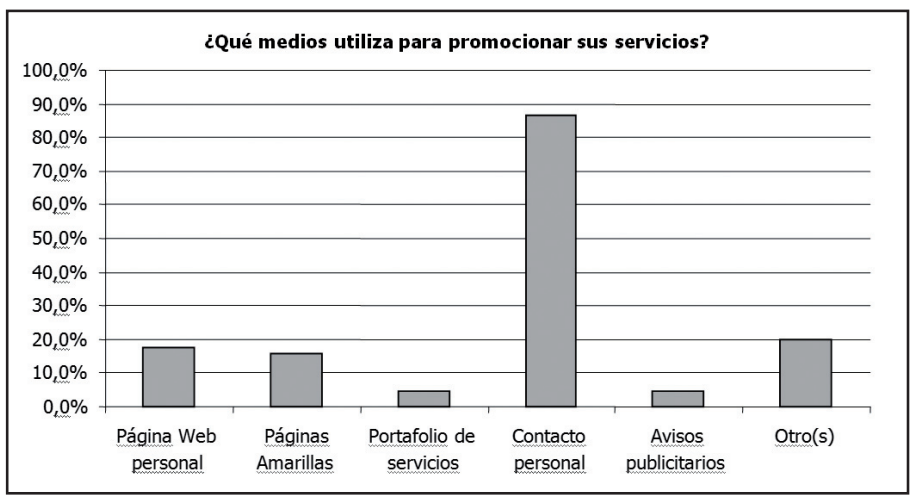

Fuente. Los autores 


\section{CONCLUSIONES}

Partiendo de los resultados de la encuesta y de las categorías empleadas para la redacción de la misma, podemos definir de forma breve hacia dónde va el perfil del traductor en Colombia.

\section{Datos personales}

Los traductores en Colombia son generalmente hombres y mujeres de nacionalidad colombiana de entre 36 y 45 años que viven principalmente en Medellín y Bogotá.

\section{Formación profesional}

Por lo general, los traductores en Colombia gozan de una buena formación profesional, ya que la mayoría de ellos estudiaron algún pregrado en traducción y además realizaron postgrados y maestrías en el mismo tema. Sin embargo, por lo general no cuentan con tarjetas profesionales que los acrediten como miembros de asociaciones de traducción importantes como la ACTI, el CCT, la ATA o la FIT.

\section{Información laboral}

Los traductores en Colombia trabajan como traductores independientes y más de la mitad se dedican a la profesión entre traductores a tiempo completo y medio tiempo.
Un buen número se dedica a la profesional de manera ocasional; incluso realizan otra actividad laboral paralela, generalmente la docencia.

Aunque son muchos los idiomas que se traducen en Colombia, por lo general se realizan traducciones directas, del inglés al español, seguido del francés al español y los tipos de textos más traducidos son los técnicos y científicos en los formatos de MS Office y PDF aunque el formato papel sigue jugando un rol importante.

\section{Aspectos profesionales}

Los traductores en Colombia tienen buenas competencias a la hora de usar un computador. Los formatos que más traducen son los de Office y las herramientas más usadas son Transit y Trados.

Respecto de la forma de cobrar, prefieren hacerlo por palabra con respecto al texto de partida si bien el cobro por página sigue siendo una práctica común.

Aunque este es el primer estudio en Colombia de este tipo, es importante resaltar que tiene sus limitaciones no solo en cuanto a la muestra sino a los resultados, como ya se ha comentado antes.

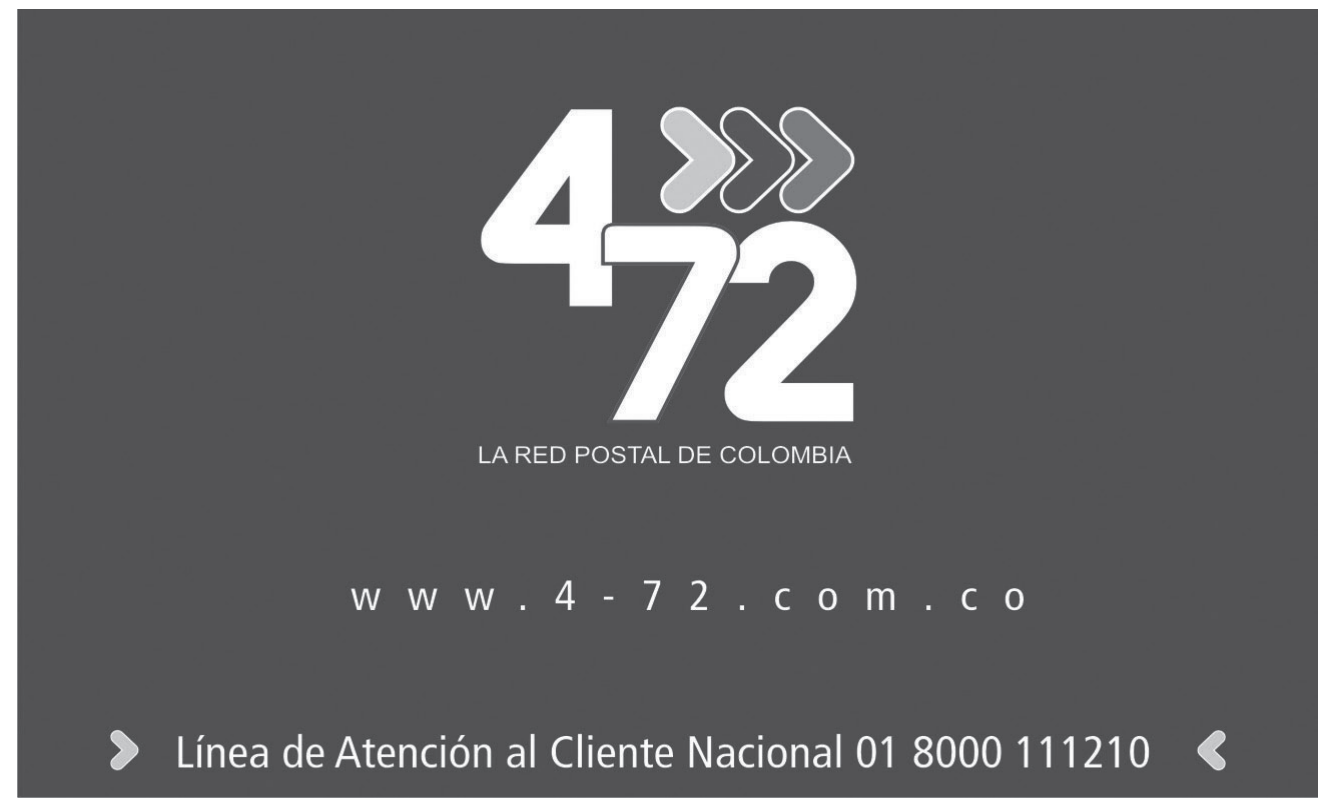




\section{REFERENCIAS BIBLIOGRÁFICAS}

Bastin, G. (1998). Latin American Tradition. En: Routledge Encyclopedia of Translation Studies. Londres: Routledge. 505-512.

Clavijo, B.; et al. (2006). Necesidad de la traducción en las empresas: procesos y perfiles. En: Revista EAN, 58. 61-78.

Dam, H. V.; Zethsen, K. (2010). Translator Status: Helpers and Opponents in the Ongoing Battle of an Emerging Profession. En: Target, 22 (2). 212-236.

Decreto 382. (1951). Cargo de Intérpretes Oficiales. Consultado en: [http://www.cntv.org.co/cntv bop/basedoc/ decreto/1951/decreto_0382_1951.html].

Flórez H. et al. (1999). Estudio de mercados Programa de Traducción. Medellín: Facultad de Ciencias Económicas, Universidad de Antioquia.

Lagoudaki, E. (2006). Translation Memory System: enlightening User's Perspectives. ASLIB International Conference 'Translating and the Computer 28', London, 15-16 November 2006.

Ley 962. (2005). Disposiciones sobre racionalización de trámites y procedimientos administrativos de los organismos y entidades del Estado y de los particulares que ejercen funciones públicas o prestan servicios públicos. Consultado en: [http://www.secretariasenado.gov.co/senado/basedoc/ley/2005/ley_0962_2005.html].

NTC 5808. (2010). Servicios de traducción. Requisitos para la prestación del servicio. Bogotá: Icontec.

Orozco, W. (2000). La traducción en el siglo XIX en Colombia. En: Íkala, 9-10. 73-88.

Quiroz, G. (2002). La terminología en Colombia: panorama general y algunas pautas históricas. En: Terminómetro, la terminología en los países andinos. 3-6.

Stejskal, J. (2005). Survey of the FIT Committee for Information on the Status of the Translation \& Interpretation Profession. Fédération Internationale des Traducteurs.

VV.AA. (1997). Libro blanco sobre la traducción en España, Madrid: ANECA Traductores.

UNE 15038. (2006). Servicios de traducción. Requisitos para la prestación del servicio.

Zielinski, D.; Ramirez, Y. (2006). Research Meets Practice: T-Survey 2005. Saarland: [S.D.]. [http://fr46.uni-saarland. de/t-survey/]. 